\title{
STI management and control in North America IUSTI region
}

\author{
Charlotte A Gaydos
}

- An additional figure is published online only. To view this file please visit the journal online (http://sti.bmj.com).

\section{Correspondence to}

Dr Charlotte A Gaydos, Division of Infectious Diseases, Johns Hopkins University, 855 North Wolfe Street, Room 530,

Rangos Building, Baltimore, MD 21205, USA;

cgaydos@jhmi.edu

Accepted 6 October 2011

\section{INTRODUCTION}

In the USA, approximately 19 million new cases of sexually transmitted infections (STIs) occur annually, ${ }_{1}^{1}$ primarily affecting adolescents and young adults. $^{2}$ Direct and indirect costs to the USA for STIs are estimated to be $\$ 10-17$ billion dollars per year (table 1). ${ }^{3}$ Issues of health inequity and health disparities make STIs a prime target for management and control. STIs present numerous barriers to routine clinical care and diagnosis because of the associated stigma, costs and confidentiality issues. Furthermore, even if tested, many infected patients $(30 \%-74 \%)$ never return for routine test results or are lost to follow-up for treatment. ${ }^{4}$

In Canada, between 1997 and 2007, both the number and rate of reported cases increased for chlamydia, gonorrhoea and infectious syphilis. ${ }^{5}$ All three STIs increased more dramatically among middle-aged adults.

\section{Age and health disparities \\ Age}

Youth of the USA represents the most vulnerable population and bears much of the burden of sexually transmitted disease (STDs). Studies indicate that one in four sexually active adolescents will acquire an STD (figure 1). The chlamydia rate in the young adolescents is astounding: $3329.3 / 100000$ and $3273.9 / 100000$ in $15-19$ and $20-24$ years women, respectively, versus 1234.0 and 511.7/100 000 for ages $25-29$ and $30-34$ years, respectively. ${ }^{2}$

In Canada, between 1997 and 2007, chlamydia rates increased by $86.8 \%$ among adults aged $15-29$ years $(p<0.0001)$ and $165.9 \%$ among adults aged 40 to 59 years old $(p<0.0001) .{ }^{5}$ Similarly, the increases for gonorrhoea were $133.3 \%(p<0.0001)$ and $210.2 \%(p<0.0001)$, respectively. Syphilis rates increased fivefold among younger adults compared with an increase of 11-fold among middle-aged adults $(p<0.0001)$ since $1997 .{ }^{5}$ The reported rate ratios (younger:middle age) decreased over time for chlamydia $(p<0.0001)$, gonorrhoea $(p<0.0001)$ and syphilis $(p=0.005)$. Men were disproportionately represented among reported chlamydia, gonorrhoea and infectious syphilis cases, constituting 59.8\%, $87.6 \%$ and $93.0 \%$ of middle-aged adult cases, respectively, in $2007 .^{5}$

\section{Race}

In the USA, STIs are a serious problem in African-Americans and ethnic minorities, ${ }^{6}$ resulting in major health disparities and inequities. ${ }^{2}$ For example, rates of infection in 2009 in black women for chlamydia are 7.8 times higher than in white women. Rates of infection in 2009 in black women for gonorrhoea are 17 times higher than in white women. Similar disproportionate burdens are noted for black men also. Primary and secondary syphilis rates show vast disparities in men (31.3 in black men vs. 3.9/100 000 in white men). ${ }^{2}$

\section{HIV risk}

Trichomonas, which is not reportable to Centers for Disease Control and Prevention (CDC), has recently been shown to be associated with HIV transmission $^{7}$ and is also associated with poor reproductive outcomes. ${ }^{8}$ Understanding the pathophysiology and epidemiology of STIs is a critical step in developing rational diagnostic, treatment and control strategies. STIs have been conclusively linked to increased risk of HIV transmission and acquisition. ${ }^{9}$ Therefore, their control and management are intrinsically linked to prevention and control of HIV.

Traditional disease control programme approaches have included clinic-based screening and partner notification. These have been supplemented by newer approaches to community-based STI control, which include use of computerised disease surveillance systems, geographic mapping and use of non-invasive new diagnostic techniques and population-based screening in the non-clinical setting. This review will cover issues of the control of STIs and their management in North America, especially as they relate to successes and failures as well as innovative new ideas of control. Areas that are paramount to understand and acknowledge, as they relate to understanding STIs and their control, include: epidemiology, behaviour, diagnosis, treatment, vaccination, education of patients and education of providers.

\section{EPIDEMIOLOGY AND BEHAVIOUR}

Understanding the epidemiology of STIs as it relates to behaviour is critical to developing effective control and management strategies. STDs are unique in the infectious disease arena, in as they are completely dependent on behavioural factors for transmission. Critical to individual patient management involves taking a good sexual risk history, yet many primary care clinicians in the USA do not even ask their patients if they are sexually active. ${ }^{10}$ In a survey of 718 patients in a adult care clinic about their provider, $44 \%$ had never been asked about sexual health by their provider, $18 \%$ had never had a gender-specific genital exam, one third had never been tested for HIV, 33\% stated they would not use a condom at next sexual act and $32 \%$ were uncomfortable discussing STIs with their provider. ${ }^{10}$ Education of primary care clinicians about STIs, their diagnosis, treatment and management is often needed. 
Table 1 Estimated annual burden and cost of STDs in the USA

\begin{tabular}{llll}
\hline Costs & $\begin{array}{l}\text { Reported } \\
\text { cases 2009 }\end{array}$ & $\begin{array}{l}\text { Estimated annual } \\
\text { new cases* }\end{array}$ & $\begin{array}{l}\text { Estimated annual } \\
\text { direct (millions) }\end{array}$ \\
\hline Chlamydia & 1244180 & 2.8 million & $\$ 701$ \\
Gonorrhoea & 301174 & 718000 & $\$ 138$ \\
HIV $\neq$ & 42959 & $60000 \S$ & $\$ 8900$ \\
Syphilis & 13997 & 70000 & $\$ 25$ \\
Hepatitis B $\neq$ & 4033 & 80000 & $\$ 47$ \\
HPV & NA & 6.2 million & $\$ 5800$ \\
Genital herpes & NA & 1.6 million & $\$ 1100$ \\
Trichomoniasis & NA & 7.4 million & $\$ 198$ \\
Total & 1606343 & 18.9 million & $\$ 17$ billion \\
\hline
\end{tabular}

*US annual estimated new cases (Weinstock et al. ${ }^{1}$ )

†Updated to 2010 \$US using medical care component of CPI. Total may differ from sum of all diseases due to rounding. Adapted from Chesson et al.

$\ddagger$ HIV and hepatitis B estimates include costs of sexually acquired cases only.

§Annual new HIV cases, 2008 estimate; all other annual cases are 2004 estimates (Hall HI,

Ruiguang $\mathrm{S}$, Rhodes $\mathrm{P}$, et al. Estimation of HIV incidence in the United States. JAMA

2009;300:520-9.).

HPV, human papillomavirus.

\section{DIAGNOSIS AND TREATMENT ISSUES}

Rapid and accurate diagnosis and correct treatment are of critical importance in the control of STIs in the USA Diagnostic approaches have been revolutionised by such techniques as nucleic acid amplification tests. These assays have simplified diagnosis because they have facilitated the use of non-invasive specimens such as urine and self-obtained vaginal swabs, which have allowed population-based surveys outside the clinic and that provide a more accurate picture of the STIs in the general population than do clinic-based surveys. ${ }^{11}{ }^{12}$ The National Health and Nutrition Examination Surveys ${ }^{1}$ have increased our knowledge of asymptomatic infections, as well as our understanding the true prevalence of STIs on a population basis. Both issues of diagnosis and treatment add knowledge as to how to design control and management strategies. Diagnostic issues that can augment control issues include the development and use of newer rapid point-of-care diagnostic tests (POCTs). These provide an immediate diagnostic result in a few minutes or within an hour. POCTs have advantages over lengthy standard diagnostic tests because they can provide an immediate diagnosis, interrupt transmission and facilitate delivery of timely prevention and counselling messages. Availability of POCTs can empower persons to ask to be tested for STIs or potentially even perform such a POCT themselves. ${ }^{13} 14$ Surveys have demonstrated that clinicians desire POCTs with specific qualities. ${ }^{15} 16$ Sadly, we have few good Federal Drug Administration-approved POC tests, ${ }^{17}$ other than for HIV and trichomonas in the USA, ${ }^{18}$ but several promising ones are in development. ${ }^{19} 20$

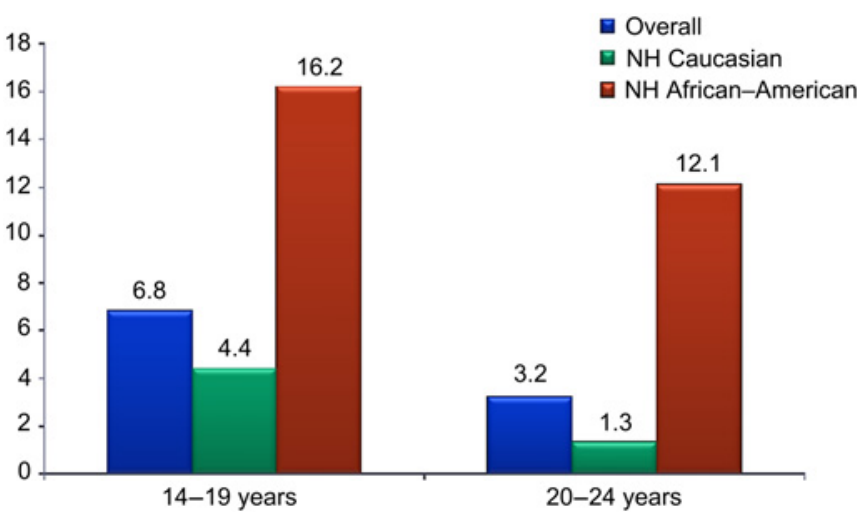

Figure 1 Chlamydia prevalence in sexually active women $14-24$ years in the USA by age and race. $\mathrm{NH}$, non-Hispanic.
Treatment has become more complex because of the emergence of antimicrobial resistance, especially for gonorrhoea, which may soon become untreatable. ${ }^{21}$ Education of providers as to the proper use of the recommended treatment strategies is paramount in preventing transmission of STIs. Treatment guidelines are issued every 2 years by the CDC to facilitate proper treatment strategies. ${ }^{22}$

\section{EDUCATION}

Since STI rates are disparate and unequal among youth and in African-Americans in North America, special education tools are needed to address the 'Hidden Epidemic'. ${ }^{23}$ Many persons, especially adolescents, and even clinicians are reluctant to discuss sexual health issues openly because of the social implications, issues of privacy and confidentiality. ${ }^{10}$ Barriers for clinicians and patients exist in getting persons tested for STIs. Focusing on sexual health rather than sexual disease is one solution to addressing these epidemics. ${ }^{24}$ However, there are barriers for both clinician sand patients to be overcome. For patients, barriers include lack of reimbursement for time required, lack of awareness that patients are sexually active and lack of knowledge that screening can be performed with non-invasive samples without a pelvic exam. For patients, barriers include inability to pay co-payments of the diagnostic test and lack of knowledge of the asymptomatic nature of STIs, high prevalence and possible adverse long-term reproductive effects of STIs.

Many clinicians are not aware that the CDC, the US Preventive Services Task Force and other professional organisations recommend screening for chlamydia in all sexually active women $<25$ years and in those $\geq 25$ years with STI risk factors. $^{2526}$ In the USA, we estimate that $<50 \%$ of all women who should be screened are being screened for chlamydia (online supplementary figure 3).

\section{BEHAVIOUR AND CONTROL INTERVENTIONS}

Assessing sexual behaviour is critical to the development of behaviour and disease control interventions. Major challenges in designing and implementing intervention strategies include using appropriate assessment tools, defining an individual's or population baseline status and implementing the intervention. In the USA, a number of large cross-sectional national surveys have been very useful in assessing behaviour and trends. These have included the National Health and Nutritional Examination Surveys, the Sexual Behaviour, Sexual Attraction and Sexual Identity in the USA: data from the 2006 to 2008 National Survey of Family Growth and the Youth Risk Behavioural Surveillance. ${ }^{12728}$ Trends in these data strongly suggest that the overall median age of sexual debut has stabilised at $15.5-16$ years. Nationwide, $46.0 \%$ of high school students had ever had sexual intercourse, and prevalence of sexual intercourse was higher in African-Americans $(65.2 \%$ vs $42.0 \%$ in Caucasians). ${ }^{28}$ By grade, ranges were $31.6 \%$ of 9 th graders to $62.3 \%$ of 12 th graders. Among $34.2 \%$ currently sexually active youth, $61.1 \%$ reported that they or their partner used a condom at the last intercourse. ${ }^{28}$ Data from the National Survey of Family Growth demonstrated that among adults aged 25-44 years, about $98 \%$ of women and $97 \%$ of men ever had vaginal intercourse, $89 \%$ of women and $90 \%$ of men ever had oral sex with an opposite-sex partner, while $36 \%$ of women and $44 \%$ of men ever had anal sex with an opposite-sex partner. More women $(13 \%)$ than men $(5.2 \%)$ reported same-sex contact in their lifetime. ${ }^{27}$ Knowledge of these behaviours is important to designing control methods.

In Canada, data from the 2005 Canadian Community Health Survey were used to examine sexual behaviour risks by geography 
and age in British Columbia. ${ }^{29}$ Outcomes investigated were ever diagnosed with an STI and did not use a condom during the last sexual intercourse. Region was categorised as metropolitan (northern, remote) and non-metropolitan (southern). In adjusted multivariate analysis, older age (25-49 years) and being female were significantly associated with previously having an STI and not using a condom during the last sexual intercourse. ${ }^{29}$ When stratified by region, the relationship between older age and not using a condom was particularly strong in northern nonmetropolitan regions. The results indicate the importance of considering older age individuals in the design of STI preventive interventions, particularly in non-metropolitan and rural and remote regions, where access to testing and treatment may be limited. ${ }^{29}$ Socioeconomic status has been linked to increased incidence of STIs in a number of different settings, including rural and urban areas in the US areas.

\section{INTERVENTIONS TO REDUCE THE STI EXPOSURE}

Promoting condom use has been one of the central tenets of the HIV and STD risk-reduction strategy in the USA. Many programmes have developed to ensure marketing and wide distribution of large numbers of condoms, and instructions for condom use have been part of the national STD guidelines since 1989. Condoms are effective when used correctly and consistently in reducing the risk of STIs, ${ }^{30}$ including HIV.

\section{BEHAVIOURAL MODELS}

When developing STD/HIV interventions specific to the North American population, behavioural models are necessary for their design and evaluation. Behavioural models are deterministic constructs of sexual behaviour. ${ }^{31}$ These constructs are usually delineated in sequential steps, with each step being (1) an environmental factor, (2) a behavioural attitude (eg, positive or negative attitude for condom use), (3) individual's area knowledge or (4) a previous behaviour. Risk behaviour is a necessary precursor step to acquisition of an STI. These contextual behavioural elements affect the outcome or the end result being a risky behaviour or a prevention behaviour.

Temporal sequence is also important to model development and interventions. The stages-of-change theory is a construct that has been used in a number of interventions, including the widely reported project RESPECT (risk-reduction counselling to prevent HIV and STDs) in the USA. ${ }^{31} 32$

\section{IMPLEMENTATION GUIDES FOR HEALTHCARE PROVIDERS}

Guides for clinicians can provide practical information and tools for encouraging providers to screen and manage patients with STIs and to assist with practical tools to enhance screening for STIs. One popular guide in the USA is 'Why screen for Chlamydia? An implementation Guide for Healthcare Providers'. ${ }^{3}$

The guide offers practical management techniques including sexual history taking, testing and counselling, treatment, patient management, and partner notification. Other resources for STI healthcare providers are available at http://www.prevent.org/ ncc and include learning materials and guides such as healthcare for adolescents; materials for professionals and tribal healers; Safe in the City, a video, intended to promote condom use; and tips for youth confidentiality.

\section{PARTNER NOTIFICATION}

Sexual partners with sexual contact with patients within 60 days should be notified to seek healthcare about exposure to an STI. Traditional partner notification methods have been used successfully previously, especially for syphilis. This has been particularly challenging in North America, especially in environments where partners are inaccessible, such as where commercial sex workers or gay men with anonymous partners are operative.

Most health departments in the USA no longer have resources for partner notification except for gonorrhoea, syphilis and HIV. Using a disease intervention, specialist is labour intensive and costly in today's shrinking budgets. Several internet accessible methods of notifying partners about exposed to an STI have become popular in the USA; most widely in use is InSPOT. ${ }^{34} 35$ Also available are CDC's e-cards (http://www2a.cdc.gov/ecards/ message/message.asp?cardid=292).

Expedited partner therapy (EPT) may be an alternative in some locations in the USA. ${ }^{36}$ In some states, it is legal for a diagnosed patient to deliver medications or a prescription directly to sexual partners without the partner being clinically assessed. EPT may be especially beneficial in situations in which the patient is doubtful a partner will seek medical care. ${ }^{36}$ The practice is growing in the USA, and it is now legal or permissible in many states. Legal status of EPT can be found at http://www. cdc.gov for each state.

\section{INNOVATIVE STI CONTROL METHODS Treatment to control transmission}

Another modality for controlling transmission of STIs has been the use of treatment regimes. Once-daily valacyclovir to reduce the risk of transmission of genital herpes has been shown to reduce transmission to partners. ${ }^{37}$ For HIV, a recent report demonstrated prevention of HIV transmission in 1763 serodiscordant couples with early antiretroviral therapy. ${ }^{38}$ Future studies will add much information for using treatment to control STIs/HIV.

\section{Vaccination to prevent human papillomavirus transmission and prevent cervical cancer}

One of our most recent and potentially most successful control programmes for STIs is the vaccine initiative to vaccinate young women against acquisition of human papillomavirus infections, thereby preventing cervical cancer. ${ }^{39} 40$ Challenges require endorsement by governments and policy makers, affordable prices and education at all levels, to overcome barriers to vaccination. ${ }^{41}$

\section{Home collection of urogenital specimens}

There has been recent interest in using samples collected at home for direct mailing to a laboratory for testing, thereby bypassing the clinic for routine screening, when an individual does not have symptoms. Although home collection is not yet Federal Drug Administration cleared, the focus of current research has shown that vaginal swabs can be collected at home and sent through the US mail to a laboratory for testing. ${ }^{42}{ }^{43}$ Two ongoing studies -I Want the Kit (IWTK) http://www.iwantthekit. org/female/default.html and I Know https://www.dontthinkknow.org/-have recruited women through specially designed websites that offer education and a home collection kit. ${ }^{44-47}$

\section{FUTURE CONSIDERATIONS}

According to the new Affordable Care Act in the USA, primary care clinicians and health directors in Federally Qualified Health Centers will be essential in the provision of routine STD and HIV testing and care. Diagnosis and treatment of STIs will shift from specialty STI clinics to primary care clinicians who will 


\section{Key messages}

- Approximately 19 million new cases of sexually transmitted infections occur annually in the USA, costing \$10-17 billion dollars a year and primarily affecting adolescents and young adults; the prevalence continues to increase.

- Sexually transmitted infections present barriers to clinical care, diagnosis and management because of the associated stigma and confidentiality issues.

- Youth, African-Americans and ethnic minorities represent the most vulnerable population and bear much of the burden of sexually transmitted infections in North America.

- Understanding the biology and epidemiology of sexually transmitted infections is a critical step in developing rational diagnostic, treatment and control strategies.

- Areas that are paramount to understand for successful control and management of sexually transmitted infections include sexual behaviour, behaviour modification models, new diagnostics, accurate treatment, vaccine availability and provider/patient education.

require training from STI clinic personnel. Since preventive services keep people healthy and reduce future healthcare costs, especially with regard to STIs and HIV, education will need to be centred on the US Federal Health Reform. The new Affordable Care Act requires Private Health Plan Coverage for Sexual Health Preventive Services. These include chlamydia tests for all sexually active women $\leq 24$ years, all pregnant women and all women at high risk who are $\geq 25$ years, as well as gonorrhoea and syphilis testing for women at risk; syphilis and HBV tests for pregnant women; and HIV tests for all high-risk adults and adolescents. Primary care clinicians in such Federally Qualified Health Centers will need training to raise awareness and acquire necessary skills to institute screening for sensitive issues such as STIs/HIV. We have much work ahead in trying to achieve better sexual health in North America. ${ }^{24}$

Competing interests None.

Provenance and peer review Commissioned; externally peer reviewed.

\section{REFERENCES}

1. Weinstock H, Berman S, Cates W Jr. Sexually transmitted disease among American youth: incidence and prevalence estimates, 2000. Perspect Sex Reprod Health 2004;36:6-10.

2. Centers for Disease Control and Prevention. Sexually Transmitted Disease Surveillance, 2009. Atlanta, GA: U.S. Department of Health and Human Services, 2010, CDC, 2010.

3. Chesson HW, Blandford JM, Gift TL, et al. The estimated direct medical cost of sexually transmitted diseases among American youth, 2000. Perspect Sex Reprod Health 2004;36:11-19.

4. Bachmann LH, Richley CM, Waites K, et al. Patterns of Chlamydia trachomatis testing and follow-up at a University Hospital Medical Center. Sex Transm Dis 1999;26:496-9.

5. Fang L, Oliver A, Jayaraman GC, et al. Trends in age disparities between younger and middle-age adults among reported rates of chlamydia, gonorrhea, and infectious syphilis infections in Canada: findings from 1997 to 2007. Sex Transm Dis 2010;37:18-25.

6. Centers for Disease Control and Prevention. CDC health disparities and inequalities report-United States, 2011. MMWR Surveill Summ 2011;60 (Suppl):1-113

7. Kissinger $\mathbf{P}$, Amadee A, Clark RA, et al. Trichomonas vaginalis treatment reduces vaginal HIV-1 shedding. Sex Transm Dis 2009;36:11-16.

8. Cotch MF, Pastorek JG 2nd, Nugent RP, et al. Trichomonas vaginalis associated with low birth weight and preterm delivery. The vaginal infections and prematurity study group. Sex Transm Dis 1997;24:353-60.

9. Fleming DT, Wasserheit JN. From epidemiological synergy to public health policy and practice: the contribution of other sexually transmitted diseases to sexual transmission of HIV infection. Sex Transm Infect 1999;75:3-17.
10. Nurutdinova D, Rao S, Shacham E, et al. STD/HIV risk among adults in the primary care setting: are we adequately addressing our patients' needs? Sex Transm Dis 2010;38:30-2.

11. Allsworth JE, Ratner JA, Peipert JF. Trichomonas and other sexually transmitted infections: results from the 2001-2004 National Health and Nutrition Examination Surveys. Sex Transm Dis 2009;36:738-44.

12. Xu F, Sternberg MR, Kottiri BJ, et al. Trends in herpes simplex virus type 1 and type 2 seroprevalence in the United States. JAMA 2006;296:964-73.

13. Huppert JS, Hesse E, Kim G, et al. Adolescent women can perform a point-of-care test for trichomonas as accurately as clinicians. Sex Transm Infect 2010;86:514-19.

14. Gaydos CA, Hsieh YH, Harvey L, et al. Will patients "opt-in" to perform their own rapid HIV test in the emergency department? Ann Emerg Med 2011;58(1 Suppl 1): S74-8.

15. Hsieh YH, Hogan MT, Barnes M, et al. Perception of an ideal point-of-care test for sexually transmitted infections - a qualitative study of eight focus group discussions. PLOS One 2010;5:e14144.

16. Hsieh YH, Gaydos CA, Hogan MT, et al. What qualities are most important to making a point of care test desirable for clinicians and others offering sexually transmitted infection testing? PLoS One 2011;6:e19263.

17. Huppert JS, Hesse E, Gaydos CA. What's the point? How point-of-care sexually transmitted infection tests can impact infected patients. Point Care 2010;9:36-46.

18. Huppert JS, Batteiger BE, Braslins P, et al. Use of an immunochromatographic assay for rapid detection of Trichomonas vaginalis in vaginal specimens. J Clin Microbiol 2005;43:684-7.

19. Pearce DM, Shenton DP, Holden J, et al. Evaluation of a novel electrochemical detection method for Chlamydia trachomatis: application for point-of-care diagnostics. IEEE Trans Biomed Eng 2011;58:755-8.

20. Zang Y, Agreda P, Kelly $\mathrm{S}$, et al. Development of a microwave-accelerated metalenhanced fluorescence 40 second, $100 \mathrm{cfu} / \mathrm{mL}$ point of care assay for the detection of Chlamydia trachomatis. IEEE Trans Biomed Eng 2011;58:781-4. Remaining References available on the web.

21. Ohnishi M, Golparian D, Shimuta K, et al. Is Neisseria gonorrhoeae initiating a future era of untreatable gonorrhea?: detailed characterization of the first strain with high-level resistance to ceftriaxone. Antimicrob Agents Chemother 2011; 55:3538-45.

22. McClelland RS, Sangare L, Hassan WM, et al. Infection with Trichomonas vaginalis increases the risk of HIV-1 acquisition. J Infect Dis 2007;195:698-702.

23. Eng TR, Butler WT. The neglected health and economic impact of STDs. In: Eng TR Butler WT, eds. The Hidden Epidemic, Confronting Sexually Transmitted Diseases. Washington, DC: National Academy Press, 1997:28-68.

24. Centers for Disease Control and Prevention. A Public Health Approach for Advancing Sexual Health in the United States: Rationale and Options for Implementation, Meeting Report of an External Consultation. Atlanta, Georgia: Centers for Disease Control and Prevention, 2010.

25. National committee for Quality Assurance. The State of Health Care Quality 2010: HEDIS Measures of Care. Washington, DC, 2010:43-4. http://www.ncqa.org/ portals/0/state\%20of\%20health\%20care/2010/SOHC\%202010\%20-\%20Full2.pdf

26. U.S.Preventive Services Task Force. Screening for chlamydial infection: U.S Preventive Services Task Force recommendation statement. Ann Intern Med 2007:147:128-34.

27. Centers for Disese Control and Prevention. Sexual behavior, sexual attraction, and sexual identity in the United States: data from the 2006-2008 National Survey of Family Growth. Natl Health Stat Report 2011;36:1-36.

28. Centers for Disease Control and Prevention, Surveillance Summaries. Sexual identity, sex of sexual contacts, and health-risk behaviors among students in grade 9 12-youth risk behavior surveillance, selected sites, United States, 2001-2009. MMWR Surveill Summ 2011;60:1-133.

29. Deering KN, Tyndal MW, Koehoorn M. Regional patterns of risk for sexually transmitted infections in British Columbia. Health Rep 2010;21:37-44.

30. Wald A, Langenberg AG, Link K, et al. Effect of condoms on reducing the transmission of herpes simplex virus type 2 from men to women. JAMA 2001;285:3100-6.

31. Zenilman JM. Behavioral interventions-rationale, measurement, and effectiveness In: Zenilman JM, Moellering RC Jr. eds. Sexually Transmitted Infections, Infect Dis Clin N Am. Philadelphia: W.B. Saunders Company, 2005:541-62.

32. Kamb ML, Fishbein M, Douglas JM Jr, et al. Efficacy of risk-reduction counseling to prevent human immunodeficiency virus and sexually transmitted diseases: a randomized controlled trial. Project RESPECT Study Group. JAMA 1998;280:1161-7.

33. Maloney SK, Johnson C. Why Screen for Chlamydia? An Implementation Guide for Healthcare Providers. Washington, DC: Partnership for Prevention, 2008:1-12.

34. Rietmeijer CA, Westergaard B, Mickiewicz TA, et al. Evaluation of an online partner notification program. Sex Transm Dis 2011;38:359-64.

35. Levine D, Woodruff AJ, Mocello AR, et al. InSPOT: the first online STD partner notification system using electronic postcards. PLoS Med 2008;5:e213.

36. Golden MR, Whittington WL, Handsfield HH, et al. Effect of expedited treatment of sex partners on recurrent or persistent gonorrhea or chlamydial infection. $N$ Engl J Med 2005;352:676-85

37. Corey L, Wald A, Patel R, et al. Once-daily valacyclovir to reduce the risk of transmission of genital herpes. N Engl J Med 2011:350:11-20.

38. Cohen MS, Chen YQ, McCauley M, et al; HPTN 052 Study Team. Prevention of HIV-1 infection with early antiretroviral therapy. N Engl J Med 2011;365:493-505. 
39. The FUTURE II Study Group. Quadrivalent vaccine against human papillomavirus to prevent high-grade cervical lesions. N Engl J Med 2007;356:1915-27.

40. Barr E, Tamms G. Quadrivalent human papillomavirus vaccine. Clin Infect Dis 2007:45:609-17.

41. Garland SM, Smith JS. Human papillomavirus vaccines: current status and future prospects. Drugs 2010;70:1079-98.

42. Cook RL, Ostergaard L, Hillier SL, et al. Home screening for sexually transmitted diseases in high risk young women: randomized controlled trial. Sex Transm Infect 2007;83:285-91.

43. Xu F, Stoner B, Taylor SN, et al. Use of home-obtained vaginal swabs to facilitate rescreening for Chlamydia trachomatis infections: two randomized controlled trials fujie. Obstet Gynecol 2011;118:231-9.
44. Gaydos CA, Barnes M, Aumakhan, et al. Characteristics of users of the Internet who submit self-obtained urogenital samples through the mail for testing sexually transmitted infections. 2008 National STD Prevention Conference; March 10-13, 2009, Chicago, IL: Abst B8b, 2008:110.

45. Gaydos CA, Barnes M, Aumakham B, et al. Can E-technology through the Internet be used as a new tool to address the Chlamydia trachomatis epidemic by home sampling and vaginal swabs? Sex Transm Dis 2009;36:577-80.

46. Gaydos CA, Hsieh YH, Barnes M, et al. Trichomonas vaginalis infection in women who submit self-collected vaginal samples after Internet recruitment. Sex Transm Dis 2011;38:828-32.

47. Kerndt P. "I Know": Combining home testing technology, social marketing, and lessions from e-commerce to fight chlamydia and gonorrhea disparities among young women. National STD Prevent Conf, Atlanta, GA, 7-11 March 2010. 